\title{
A Case of Overlap Syndrome of Systemic Sclerosis and Cryoglobulinemic Vasculitis With Central Nervous System Involvement
}

\author{
Youjin Jung, M.D. ${ }^{1}$, Eunyoung Emily Lee, M.D. ${ }^{2}$, Jina Yeo, M.D. ${ }^{1}$, Mi Hyeon Kim, M.D. ${ }^{1}$, Se Rim Choi, M.D. ${ }^{1}$, \\ Yeong Wook Song, M.D., Ph.D. ${ }^{1}$ \\ ${ }^{1}$ Division of Rheumatology, Department of Internal Medicine, Seoul National University College of Medicine, Seoul, ${ }^{2}$ Division of Rheumatology, \\ Department of Internal Medicine, Uijeongbu Eulji Medical Center, Eulji University School of Medicine, Uijeongbu, Korea
}

\begin{abstract}
Mixed cryoglobulinemic vasculitis (CV) is occasionally caused by autoimmune diseases including systemic sclerosis. Multiorgan involvement such as skin, kidney, and peripheral nerve involvement is common in mixed CV. However, central nervous system (CNS) involvement is extremely rare. Here, we report a case of overlap syndrome of limited cutaneous systemic sclerosis and mixed cryoglobulinemic vasculitis with CNS involvement. The neurologic deficits and systemic symptoms improved promptly after steroid and cyclophosphamide therapy. (J Rheum Dis 2021;28:242-246)
\end{abstract}

Key Words. Cryoglobulinemia, Vasculitis, Central nervous system, Scleroderma, systemic

\section{INTRODUCTION}

Cryoglobulins (CGs) are immune complexes that precipitate from serum at temperatures below $37^{\circ} \mathrm{C}$ and redissolve upon rewarming. There are three different subgroups of CGs based on their composition. Type I shows an isolated monoclonal immunoglobulin; type II includes polyclonal IgGs and monoclonal IgM with rheumatoid factor (RF) activity; type III includes polyclonal IgGs and polyclonal IgMs with RF activity [1]. Type II and III CGs also refer to mixed cryoglobulins. Cryoglobulins are not pathogenic in most cases but may lead to small-to-medium vessel vasculitis called cryoglobulinemic vasculitis (CV) in $2 \% \sim 50 \%$ of the cases [2].

Type I CV is commonly found in patients with lymphoproliferative disorders and represents the symptoms secondary to vascular occlusion including Raynaud's phenomenon, ischemic ulcers, and gangrene. When the cryocrit is high, symptoms of hyperviscosity may occur such as blurred vision, headache, and other symptoms related to encephalopathy [3]. Mixed CV is associated with infectious disease, notably chronic hepatitis $\mathrm{C}$ infection, and autoimmune diseases such as Sjögren's syndrome and systemic lupus erythematosus [2]. In addition, there have been several case reports of overlap syndrome of systemic sclerosis (SSc) and cryoglobulinemic vasculitis [4]. In mixed $\mathrm{CV}$, nonspecific symptoms such as myalgia, fatigue, and arthralgia are common, and cutaneous vasculitis including purpura and peripheral nerve involvement is more frequent [5]. However, central nervous system (CNS) involvement was only seen in $0 \% \sim 3 \%$ of the patients in several large case series $[3,6]$.

We report a rare case of CNS involvement in cryoglobulinemic vasculitis in a patient with limited cutaneous SSc.

\section{CASE REPORT}

A 41-year-old female patient visited our hospital due to the sudden onset of visual disturbances. She had been di-

Received : February 15, 2021, Revised : March 19, 2021, Accepted : March 28, 2021

Corresponding to : Yeong Wook Song (D)http://orcid.org/0000-0002-5384-3437

Division of Rheumatology, Department of Internal Medicine, Seoul National University College of Medicine, 101 Daehak-ro, Jongno-gu, Seoul 03080, Korea. E-mail : ysong@snu.ac.kr 
agnosed with limited cutaneous SSc three years earlier when she presented with manifestations including skin sclerosis of the finger, hand, forearm, and face. She had a fish-mouth appearance, Raynaud's phenomenon, digital ulcer, multiple telangiectasias on the face, reflux esophagitis, and interstitial lung disease. She had diffusely reduced nail-fold capillaries with bulging and microhemorrhages. A skin biopsy of the forearm showed increased dermal collagen deposition with superficial perivascular lymphocytic infiltration. The fluorescent antinuclear antibody test was positive $(>1: 320$, nucleolar pattern). And anti-centromere antibody and anti-Scl-70 antibody test were negative. She fulfilled the American College of Rheumatology/European League Against Rheumatism 2013 criteria for the classification of SSc. She had been treated with bosentan and aspirin for Raynaud's phenomenon and the digital ulcer. There was no evidence of progression of interstitial lung disease on the two-year follow-up chest computerized tomography and pulmonary function test.

Seven days before visiting our hospital, the patient was admitted to another hospital due to a fever of over $38^{\circ} \mathrm{C}$ and a headache. The cerebrospinal fluid (CSF) analysis showed protein $161.6 \mathrm{mg} / \mathrm{dL}$, glucose $39 \mathrm{mg} / \mathrm{dL}$, red blood cells (RBCs) $0 / \mathrm{mm}^{3}$, and white blood cells (WBCs) $459 / \mathrm{mm}^{3}$ (88\% polymorphonuclear cells). The serum C-reactive protein (CRP) and erythrocyte sedimentation rate (ESR) were $10.6 \mathrm{mg} / \mathrm{dL}$ and $82 \mathrm{~mm} / \mathrm{h}$, respectively. Magnetic resonance imaging (MRI) of the brain showed several T2 hyperintense lesions on the frontal, left parietal, and left occipital lobes and prominent leptomeningeal enhancement. The initial assessment was bacterial meningitis with early encephalitis, and empirical antibiotic therapy (ceftriaxone and vancomycin) was started. However, visual disturbances developed after four days of treatment with antibiotics. The follow-up MRI of the brain showed increased size and number of multifocal T2 hyperintense lesions along the bilateral cerebral hemisphere and midbrain including a 4.5-cm-sized lesion on the right posterior lobe (Figure 1). The results of CSF Gram staining and culture, PCR test for herpes simplex virus and enteroviruses, cryptococcus antigen test, and fungal culture were all negative. She was referred to our hospital for further evaluation.

The patient had visual impairment to the extent of counting the number of fingers, and also complained of headache, dizziness, fever, myalgia, polyarthralgia, and a tingling sensation in both feet. Her blood pressure (BP) was 192/103 mmHg, heart rate was $48 / \mathrm{min}$, and body temperature was $37.3^{\circ} \mathrm{C}$. The electrocardiogram revealed sinus bradycardia with normal QT interval. The neurological examination showed a drowsy mental state and neck stiffness. The sensory examination revealed paresthesia in both feet and the muscle strength examination showed bilateral symmetric weakness (4/5) of the upper and lower extremities. The deep tendon reflex was positive in the biceps tendon, negative in the patella tendon, and the Babinski sign was positive bilaterally. There was
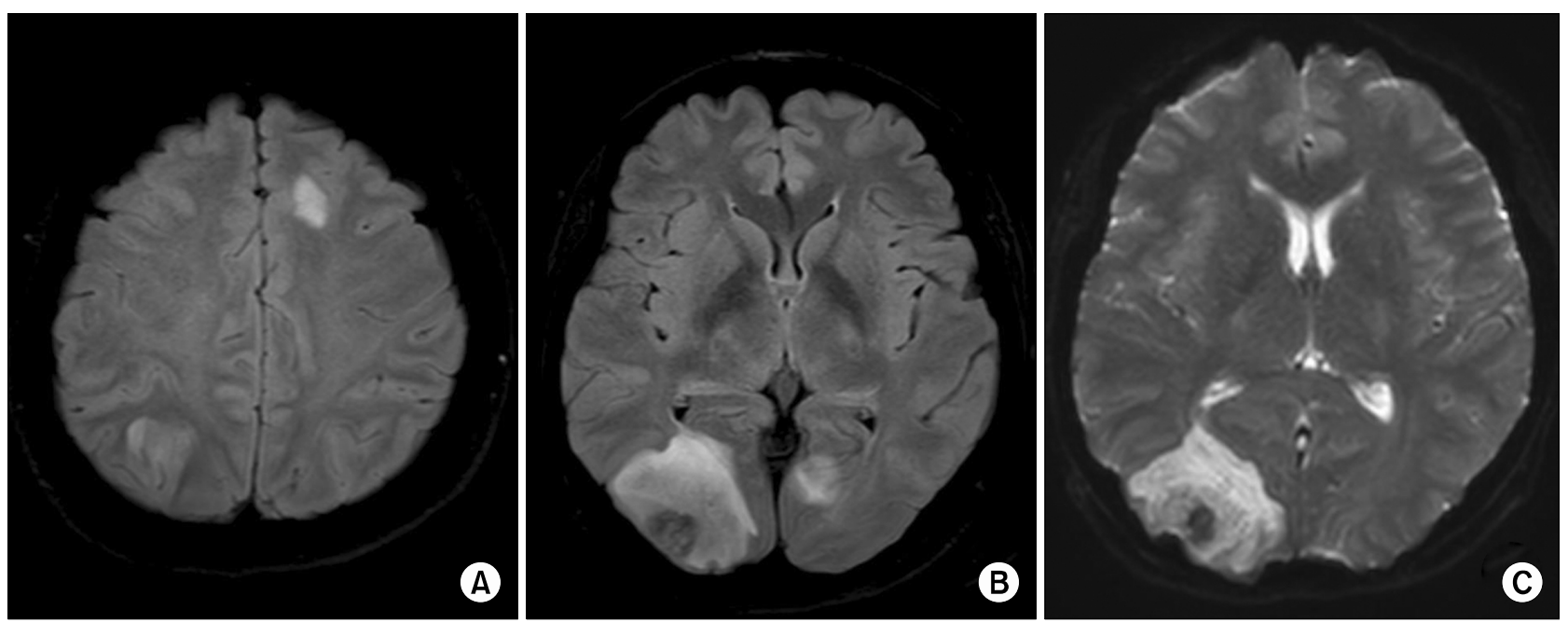

Figure 1. Brain MRI shows a number of multifocal patchy $T 2$ high signal intensity areas in the bilateral cerebral hemisphere (A) and a 4.5-cm-sized lesion with inner hemorrhage with subtle enhancement on the right posterior lobe in the T2 FLAIR (B) and DWI images (C). MRI: magnetic resonance imaging, FLAIR: fluid attenuated inversion recovery, DWI: diffusion-weighted imaging. 
a skin ulcer $(4 \times 5 \mathrm{~cm})$ on the right lateral malleolar area and no evidence of synovitis in the peripheral joints. The WBC count was $14,090 / \mathrm{mm}^{3}$, the CRP was $3.09 \mathrm{mg} / \mathrm{dL}$, and the ESR was $56 \mathrm{~mm} / \mathrm{h}$. Antibiotic therapy was continued and intravenous acyclovir, nicardipine, and mannitol, and dexamethasone treatment for brain edema were started. Her BP stabilized within six hours. However, visual impairment deteriorated, and left-sided weakness and Raynaud's phenomenon developed in fingers.

The emergency cerebral angiography showed diffuse stenosis and a beaded pattern of both the anterior and middle cerebral arteries and their branches, and diffuse luminal narrowing with dysplastic changes in the right distal vertebral artery and basilar artery trunk (Figure 2). The impression was cerebral angiitis. Therefore, methylprednisolone 1,000 mg pulse therapy was started and continued for three days. On the second day of corticosteroid administration, the patient displayed a gradual improvement in her mental status, and fever resolved. Three days later, she was able to read the text on a nameplate, and the motor function markedly improved. Four days after the end of the corticosteroid pulse treatment, the level of ESR and CRP were $15 \mathrm{~mm} / \mathrm{h}$ and $0.29 \mathrm{mg} / \mathrm{dL}$, respectively. The follow up angiography was not performed. But, ten days after the end of the corticosteroid pulse treatment, CSF analysis showed protein $40 \mathrm{mg} / \mathrm{dL}$, RBCs $0 / \mathrm{mm}^{3}$, and $\mathrm{WBCs} 3 / \mathrm{mm}^{3}$. The laboratory results showed positive cryoglobulins, markedly reduced C4 levels $(<1$ $\mathrm{mg} / \mathrm{dL}$ ), and positive rheumatoid factor $(15 \mathrm{IU} / \mathrm{mL})$. Anti-hepatitis C virus antibody and hepatitis B surface antigen were negative. The results of anti-neutrophil cytoplasmic antibody, anti-double-stranded DNA antibody, anti-Sm antibody, anti-phospholipid antibody including lupus anticoagulant, anti-cardiolipin IgG and IgM, and anti-beta2 glycoprotein I IgG and IgM, and venereal disease research laboratory (VDRL) test were all negative. Serum immunofixation electrophoresis revealed monoclonal gammopathy (IgM and kappa type), and there was no evidence of clonal plasma cell infiltration on the bone marrow examination. And the positron emission tomography (PET) scan was normal. The nerve conduction velocity test revealed sensory-motor polyneuropathy of both feet. The clinical diagnosis was cryoglobulinemic vasculitis with CNS involvement and we additionally administrated intravenous cyclophosphamide $750 \mathrm{mg}$. She has been followed in the outpatient clinic without a flare-up for four months.

\section{DISCUSSION}

To the best of our knowledge, this was the first case of
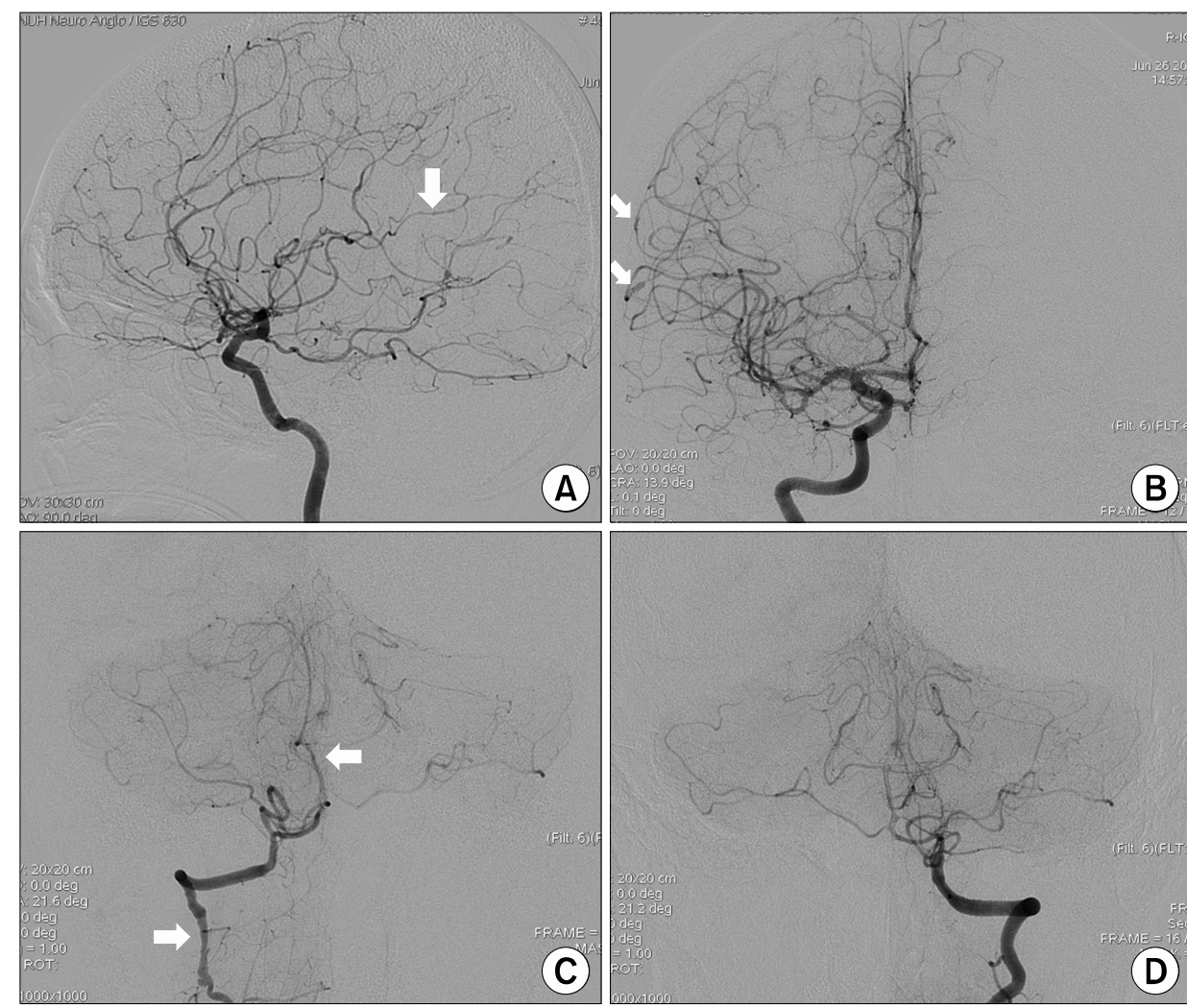

C)

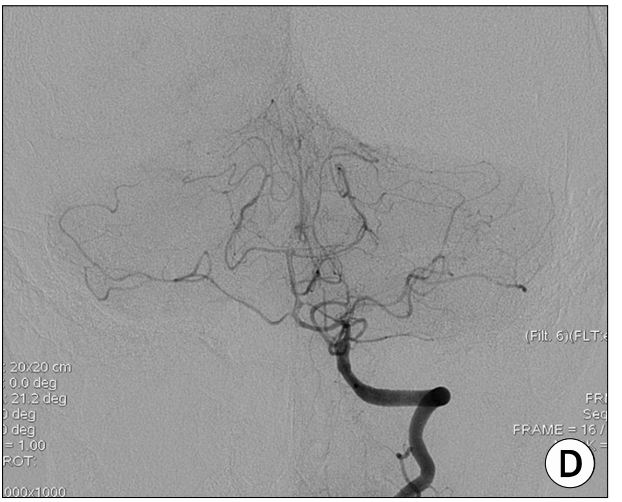

Figure 2. Lateral view (A) and anterior-posterior view (B) of the conventional cerebral angiography shows diffuse stenosis and beaded pattern (white arrows) of the anterior and middle cerebral arteries and their branches. The right distal vertebral artery and basilar artery trunk show diffuse luminal narrowing with dysplastic changes (white arrows) (C) compared to the left side (D). 
overlap syndrome of SSc and cryoglobulinemic vasculitis with CNS involvement. Cryoglobulinemic vasculitis and SSc often manifest as skin ulcers and peripheral neuropathy $[4,7]$. In one study, four out of 246 (1.6\%) SSc patients were diagnosed with mixed $\mathrm{CV}$ and shared a number of manifestations. All patients had limited cutaneous SSc with serum anticentromere antibodies, complicated by severe and non-healing skin ulcers of the lower limbs, peripheral neuropathy, and pulmonary hypertension, but no one presented with CNS involvement [4].

Cryoglobulinemic disease is diagnosed by the presence of typical organ involvement (mainly skin, kidney, or peripheral nerves) and circulating CGs in most cases [8]. In this case, the patient presented with constitutional symptoms (fever and fatigue), arthralgia, Raynaud's phenomenon, skin ulcer, peripheral neuropathy, and CNS vasculitis. The laboratory findings supporting the diagnosis were positive cryoglobulin and rheumatoid factor tests, and reduced C4 levels. We presumed it was type 2 cryoglobulinemic vasculitis because the lab results showed low $\mathrm{C} 4$ levels, rheumatoid factor activity, and IgM and kappa type monoclonal gammopathy on immunofixation serum electrophoresis [9]. Furthermore, there was no evidence of lymphoproliferative disease in the bone marrow examination and the PET scan in the present case.

The initial CSF analysis showed high protein and low glucose levels, and neutrophilic pleocytosis. These findings suggested the possibility of infectious meningitis. However, there were no isolated bacterial, viral, and fungal pathogen in the CSF analysis. In addition, after administering seven days of empirical antibiotics and five days of antiviral agents, the patient had sustained fever and neurological deterioration. Fever and the neurological deficits were relieved within three days after treatment with a high-dose corticosteroid. In several case series, patients with cryoglobulinemic vasculitis and CNS manifestations had normal [10] or abnormal CSF values including elevated protein concentrations or lymphocytic pleocytosis [11]. However, neutrophilic pleocytosis was also reported in a case of CV with CNS involvement [12].

A brain biopsy was not performed in the present case. However, there were a number of findings supporting the diagnosis of CNS vasculitis, such as pleocytosis in the CSF, typical conventional cerebral angiographic findings including multiple segmental narrowing (beaded-pattern) of the cerebral arteries, and multiple T2 high signal intensity lesions in the bilateral hemispheres on brain MRI [13]. Most of the neurologic deficits and constitu- tional symptoms were relieved after treatment with high-dose corticosteroid therapy. The main differential diagnosis was reversible posterior leukoencephalopathy syndrome (PRES). The clinical symptoms of PRES include encephalopathy $(50 \% \sim 80 \%)$, headache $(50 \%)$, visual disturbances (33\%), and focal neurologic deficits (10\% 15\%), and are mainly caused by high blood pressure. Brain imaging usually reveals vasogenic edema, which appears as dark lesions in diffusion-weighted imaging (DWI) in the parieto-occipital regions of both cerebral hemispheres [14]. However, in this case, the brain MRI showed multifocal hyperintense lesions on T2/FLAIR including unilateral cytotoxic edema (high signal on DWI) affecting solely the posterior lobe. In addition, the hypertensive crisis generally precedes neurologic syndrome in PRES [15]. However, the patient in the present case presented with neurologic manifestations prior to BP elevation.

\section{SUMMARY}

We report a case of overlap syndrome of limited cutaneous SSc and mixed cryoglobulinemic vasculitis with CNS involvement. The symptoms improved promptly after corticosteroid and cyclophosphamide therapy.

\section{CONFLICT OF INTEREST}

No potential conflict of interest relevant to this article was reported.

\section{AUTHOR CONTRIBUTIONS}

Study concept and design: Y.J., Y.W.S. Acquisition of data: Y.J., E.E.L., J.Y., Y.W.S. Analysis and interpretation of data: Y.J., E.E.L., Y.W.S. Interpretation of the imaging data: Y.J., M.H.K., S.R.C., Y.W.S. Writing manuscript: Y.J., Y.W.S. All authors read and approved the final manuscript.

\section{REFERENCES}

1. Brouet JC, Clauvel JP, Danon F, Klein M, Seligmann M. Biologic and clinical significance of cryoglobulins. A report of 86 cases. Am J Med 1974;57:775-88.

2. Trejo O, Ramos-Casals M, García-Carrasco M, Yagüe J, Jiménez S, de la Red G, et al. Cryoglobulinemia: study of etiologic factors and clinical and immunologic features in 443 patients from a single center. Medicine (Baltimore) 
2001;80:252-62.

3. Terrier B, Karras A, Kahn JE, Le Guenno G, Marie I, Benarous L, et al. The spectrum of type I cryoglobulinemia vasculitis: new insights based on 64 cases. Medicine (Baltimore) 2013;92:61-8.

4. Giuggioli D, Manfredi A, Colaci M, Manzini CU, Antonelli A, Ferri C. Systemic sclerosis and cryoglobulinemia: our experience with overlapping syndrome of scleroderma and severe cryoglobulinemic vasculitis and review of the literature. Autoimmun Rev 2013;12:1058-63.

5. Ramos-Casals M, Trejo O, García-Carrasco M, Cervera R, Font J. Mixed cryoglobulinemia: new concepts. Lupus 2000; 9:83-91.

6. Terrier B, Krastinova E, Marie I, Launay D, Lacraz A, Belenotti P, et al. Management of noninfectious mixed cryoglobulinemia vasculitis: data from 242 cases included in the CryoVas survey. Blood 2012;119:5996-6004.

7. Quéméneur T, Mouthon L, Cacoub P, Meyer O, MichonPasturel U, Vanhille P, et al. Systemic vasculitis during the course of systemic sclerosis: report of 12 cases and review of the literature. Medicine (Baltimore) 2013;92:1-9.

8. Silva F, Pinto C, Barbosa A, Borges T, Dias C, Almeida J. New insights in cryoglobulinemic vasculitis. J Autoimmun
2019;105:102313.

9. Desbois AC, Cacoub P, Saadoun D. Cryoglobulinemia: an update in 2019. Joint Bone Spine 2019;86:707-13.

10. Petty GW, Duffy J, Houston J 3rd. Cerebral ischemia in patients with hepatitis $\mathrm{C}$ virus infection and mixed cryoglobulinemia. Mayo Clin Proc 1996;71:671-8.

11. Feldman L, Dhamne M, Li Y. Neurologic manifestations associated with cryoglobulinemia: a single center experience. J Neurol Sci 2019;398:121-7.

12. Ristow SC, Griner PF, Abraham GN, Shoulson I. Reversal of systemic manifestations of cryoglobulinemia. Treatment with melphalan and prednisone. Arch Intern Med 1976;136: 467-70.

13. Byram K, Hajj-Ali RA, Calabrese L. CNS vasculitis: an approach to differential diagnosis and management. Curr Rheumatol Rep 2018;20:37.

14. Fugate JE, Rabinstein AA. Posterior reversible encephalopathy syndrome: clinical and radiological manifestations, pathophysiology, and outstanding questions. Lancet Neurol 2015;14:914-25.

15. Stott VL, Hurrell MA, Anderson TJ. Reversible posterior leukoencephalopathy syndrome: a misnomer reviewed. Intern Med J 2005;35:83-90. 